
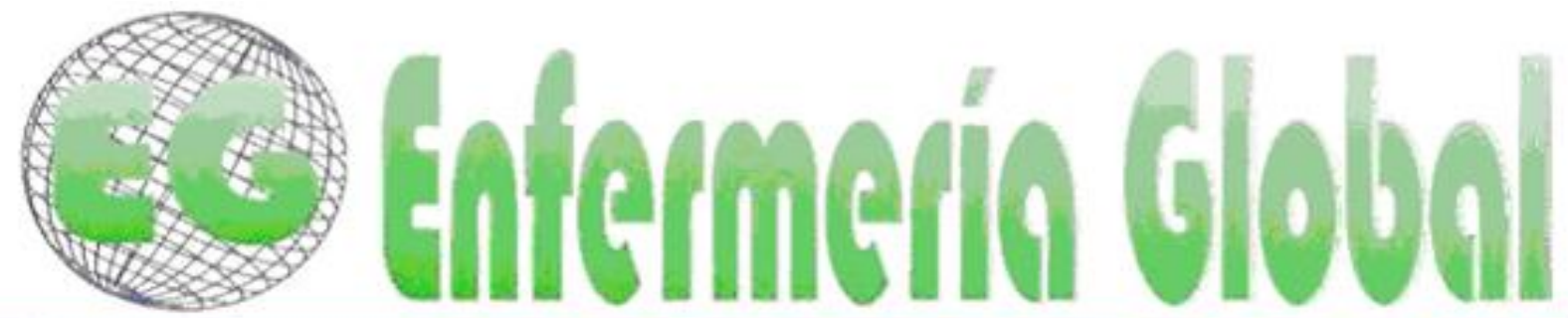

$N^{\circ} 33$

\title{
Modelo evaluativo del costo fármaco hospitalario de pacientes con enfermedades respiratorias del Hospital Rafael González Plaza de Naguanagua estado Carabobo
}

Evaluative model of the medical pharmaceuticals costs in patients who suffer breathing diseases at the Rafael González Plaza hospital from Naguanagua, Carabobo state.

\author{
*Petip, Sor Elena **Flores, Damary \\ *Licenciada en Enfermería, con Post Grado en Enfermería Comunitaria. Docente contratado. E-mail: \\ elenapetit@hotmail.com ** Profesora. Universidad Rômulo Gallegos. Venezuela.
}

Palabras clave: Modelo; Costo; Fármacohospitalario; enfermedades respiratorias.

Keywords: Model, Cost Fármacohospitalario; respiratory diseases.

\section{RESUMEN}

En el Hospital Dr. Rafael González Plaza, los pacientes ingresados con enfermedades respiratorias permanecen en el centro de salud un lapso que supera las expectativas médicas. De allí que surge el interés de realizar una investigación con el objetivo de proponer un modelo evaluativo del costo Fármaco hospitalario de pacientes con Enfermedades respiratorias que ingresan al Hospital Rafael González Plaza de Valencia, Estado Carabobo, en el primer trimestre del año 2008. La investigación planteada corresponde al diseño de investigación no experimental, enmarcada dentro de la investigación de campo, modalidad proyecto factible. La población y muestra 20 pacientes hospitalizados en el servicio de hombres y 12 miembros del personal administrativo. Se aplicaron dos instrumentos. El primero conformado por dos partes: La primera, con los datos demográficos de los encuestados, consta de 4 ítems. La segunda, conformada por seis ítems referidos al costo farmacológico. Consta de 6 ítems con alternativas de respuesta policotómica. El segundo cuestionario, está conformado por siete preguntas con alternativas de respuesta cerradas, aplicado a los miembros del personal administrativo. La validez se obtuvo a través de juicio de expertos en el área de enfermería. La confiabilidad, por medio de la fórmula Alfa de Cromback, dio como resultado un índice de 0,82. Los resultados son contrastantes para ambos estratos, ya que al tratar de establecer comparación entre las respuestas emitidas por los sujetos, a juicio de los usuarios, no se realizan acciones dirigidas a estimar el costo farmacológico, mientras que el personal administrativo, refiere que sí. Se recomienda el diseño del modelo evaluativo del costo fármaco Hospitalario.

\section{ABSTRACT}

At Dr. Rafael González Plaza hospital, the patients with breathing diseases stay in the health service more time than the expected by the doctors. From that, an interest to carry out a research aimed at the proposal of an evaluative model of the medical pharmaceuticals costs in patients who suffer breathing 
diseases that goes to Rafael González Plaza de Valencia hospital in Carabobo state during the first trimester of the year 2008. The set out research corresponds to the design of the non-experimental research, framed in the field research, modality of viable project. The sample population was 20 hospitalized patients in the men service and 12 administrative staff members. Two tools were applied. The first formed by two parts: the first, with the demographic data of the respondents, has 4 items. The second formed by 6 items referred to the pharmacological cost, is formed by 6 items with alternatives of multiple answers. The second questionnaire is formed by seven questions with closed alternative answers, applied to the administrative staff. The validity was reached by means of the experts' opinions in the field of nursing. The confidentiality, by means of the Alfa de Cromback formula, gave as a result un index of 0,82 . The results are contrasted to both stratus, as trying to establish comparison between the emitted answers by the subjects, as far as the users opinions is concerned, actions directed to estimate the pharmacological costs are not carried out, while the administrative staff, refers the opposite. It is recommended the design of an evaluative model of the medical pharmaceuticals costs.

\section{INTRODUCCIÓN}

Las enfermedades respiratorias constituyen una causa común de morbi-mortalidad, forma parte de un grupo de pacientes que padecen Enfermedad Pulmonar Obstructiva Crónica (EPOC) y en los Estados Unidos cerca de 10 millones de personas sufren de este proceso. Actualmente, es la quinta causa de muerte, su incidencia se ha incrementado paulatinamente desde 1979 a 1989 hasta un 69\%, y la mortalidad también se incrementó en ese mismo período en un $52 \%{ }^{(1)}$.

Esta enfermedad junto con la neumonía, el enfisema y el asma ocupó para el año 2006 el 16o. lugar entre las principales causas de morbilidad hospitalaria en el Sistema Nacional de Salud, con una tasa de 97.6 por 10.000 habitantes. La mortalidad general por estas mismas causas ocupó el 17o. lugar, con una tasa de 11.2 por 100.000 habitantes 12 para el mismo año. Es posible esperar que este número de casos se encuentre subestimado debido a que la sintomatología de las enfermedades respiratorias no implica, en todos los casos, demanda de atención médica ${ }^{(2)}$.

Las repercusiones de esta enfermedad específica, representan costos que no se limitan a un sólo sector. Afectan de manera diferencial a los grupos que conforman la sociedad y prácticamente a la totalidad de los aspectos de la vida económica y social. Sin embargo, los costos en salud usualmente son valorados a partir de las erogaciones realizadas por los servicios, y sólo han sido explorados parcialmente en los otros ámbitos ${ }^{(3)}$.

Así, las experiencias realizadas en Latinoamérica indican que la metodología usual para estimar los costos asociados a una patología es recoger parte de los gastos directos del sector doméstico en encuestas de ingreso-gasto y en algunas encuestas del sector. Se ha identificado que el gasto en salud en los hogares es proporcionalmente tan grande como el gasto público a nivel agregado. Los gastos indirectos que generan las enfermedades crónicas, generalmente no se han registrado en Venezuela, y hasta ahora no existe información sobre su magnitud. En este sentido, los trastornos respiratorios irán en aumento en la población, el fenómeno de la inmigración, que conlleva un potencial de marginación, la falta de mantenimiento en filtros de aire acondicionado y el tabaquismo activo y pasivo, que en la actualidad es responsable de un $25 \%$ de las muertes prematuras, serán factores favorecedores de la prevalencia de las patologías respiratorias. Las perspectivas futuras también indican que factores como el urbanismo creciente, el estilo de vida acelerado, el 
cambio climático y otros factores medioambientales, se asocian a un aumento de enfermedades respiratorias, con el consecuente costo social de las mismas ${ }^{(3)}$.

Entre estos costos, figuran: "los días productivos perdidos del paciente y su acompañante, modificaciones en la vivienda por la enfermedad, entre otras" (1). La experiencia obtenida en otros países como Costa Rica, México y Chile, ha documentado que estos costos son aún más elevados que los que representan la atención del paciente en los servicios de salud.

Según refiere el autor citado "Los virus respiratorios son reflejo, generalmente, de su epidemiología en la comunidad, existiendo infecciones por virus respiratorio sincicial (VRS), virus parainfluenza, virus influenza, adenovirus y bacterias como Streptococcus pneumoniae, Haemophilus influenzae tipo b, Neisseria meningitidis y Streptococcus. pyogenes" ${ }^{(1)}$. De allí que su transmisión es por inoculación directa de partículas infecciosas desde otros enfermos, sin olvidar por ejemplo el riesgo de transmisión de Bordetella pertussis desde adultos asintomáticos a lactantes no vacunados. En este grupo erario, existen elevadas tasas de portación de bacterias potencialmente patógenas entre 3 meses y 4 años de edad, con una frecuencia de $60,2 \%$ y resistencia a penicilina en $28,4 \%$ de las cepas aisladas. Es por ello, que la mayor parte de los padecimientos, sobre todo los crónico-degenerativos e incapacitantes, generan alteraciones en la calidad de vida y requieren tratamiento hospitalario con un elevado costo farmacológico, que por lo general no se expresan monetariamente. El propósito de realizar esta estimación es identificar una dimensión cuantitativa que resulte comparable con el costo de las otras dimensiones del daño por una enfermedad.

En el Estado Carabobo, la situación es similar al resto del país. Según Epidemiología Regional, la atención de enfermos respiratorios crónicos se está convirtiendo en un asunto de primer orden. En los últimos años pocas enfermedades han supuesto un problema de salud laboral equiparable al asma. Se espera que para el año 2009, se una a ellos un alto índice apnea del sueño y de enfermedades pulmonares de base inmunológica. Esto se debe a que en las empresas, los sistemas de aire son el caldo de cultivo perfecto para la reproducción de contaminantes biológicos, debido a sus espacios cerrados, temperatura constante, humedad y suciedad como nutrientes que con el paso del tiempo acumulan polvo, suciedad, bacterias, hongos, mohos y hasta roedores y cucarachas muertas. Lo cual hace que al salir el aire este altamente contaminado. ${ }^{(3)}$

En cuanto a la situación, cabe destacar que en el Hospital Dr. Rafael González Plaza, institución dependiente del Ministerio del Poder Popular para la Salud y Desarrollo Social, el cual tiene como función contribuir con otros establecimientos de salud a mejorar la calidad de vida de la población local, en lo referente a enfermedades respiratorias. En este sentido, las estadísticas de dicho centro asistencial revelan un ingreso de usuarios con enfermedades respiratorias, que según estadísticas de epidemiología Regional (2007), se han venido incrementado en los últimos cinco años. Para este año, se atendieron 252 casos, de los cuales un 50\% (128 casos) obedeció a enfermedades respiratorias, el 30\% (77 casos) a enfisemas pulmonar y el restante $20 \%$ (51 casos) a broncoestasias.

El presente estudio tiene como Objetivo General: Proponer un modelo evaluativo del costo Fármaco hospitalario de pacientes con Enfermedades respiratorias que 
ingresan al Hospital Rafael González Plaza de Valencia Estado Carabobo en el primer trimestre del año 2008.

\section{Los objetivos específicos son los siguientes:}

1. Describir las características sociodemográficas de los pacientes con Enfermedades respiratorias que ingresan al Hospital DR. Rafael González Plaza.

2. Determinar el costo fármaco hospitalario de pacientes que ingresan con enfermedades respiratorias en el Hospital DR. Rafael González Plaza

3. Configurar los elementos de un modelo evaluativo del costo hospitalario de de los pacientes con Enfermedades respiratorias que ingresan al Hospital DR. Rafael González Plaza.

4. Diseñar un modelo evaluativo del costo fármaco hospitalario de los pacientes con Enfermedades respiratorias que ingresan al Hospital DR. Rafael González Plaza.

Como aporte científico, se espera de esta investigación, que la cuantificación de los costos por padecimiento cobre relevancia debido a que el incremento en la esperanza de vida de la población en Venezuela conlleva la existencia de un mayor número de enfermos; y la disponibilidad de tecnologías crecientemente complejas y sofisticadas se traduce en el incremento en los costos de la atención. Al diseñar un modelo evaluativo del costo hospitalario, se encuentra la posibilidad de visualizar de manera global la funcionalidad del sistema sanitario del país, lo que permitirá establecer los correctivos necesarios para lograr la meta de salud para todos.

\section{MATERIAL Y MÉTODOS}

El trabajo corresponde a una investigación no experimental, de campo ya que los datos se tomaron directamente de la realidad, Respecto a la investigación de Campo, es aquella en que "el mismo objeto de estudio sirve como fuente de información para el investigador" (4)

La población se define como "la totalidad del fenómeno a estudiar en donde las unidades de población poseen unas características comunes, la cual se estudia y da origen a los datos de investigación" ${ }^{(5)}$. La población objeto de estudio y sobre la cual se generalizan los resultados estuvo conformada por dos estratos: 20 pacientes hospitalizados en el servicio de hombres y 12 miembros del personal administrativo. En cuanto a la muestra seleccionada para efectos de la investigación, la muestra se consideró igual a la población por ser finita y censal, es decir, estará conformado por el $100 \%$ de la población, es decir, 12 miembros del personal y 20 pacientes del servicio de hombres.

Para recolectar la información necesaria se elaboraron dos cuestionarios que permitirán obtener la información de la muestra. Para el diseño se tomó en cuenta la relación existente entre los objetivos de la investigación, las variables, las dimensiones y los indicadores involucrados en el estudio. El primer instrumento, fue un cuestionario conformado por dos partes: La primera, con los datos demográficos de los encuestados, consta de 4 ítems. La segunda, conformada por seis ítems 
referidos al costo farmacológico. Consta de 6 ítems con alternativas de respuesta Siempre, Casi siempre y Nunca. El segundo cuestionario, estuvo conformado por siete preguntas con alternativas de respuesta cerradas, que será aplicado a los miembros del personal administrativo

Para la validez, se sometió el instrumento a un juicio de tres (3) expertos, estos serán los que tendrán la responsabilidad de emitir su opinión en cuanto a la pertinencia entre el instrumento que se aplicará y la investigación. A cada uno de los expertos se les entregará la ficha de recolección de datos diseñada, la tabla de operacionalización de variables así como también título del trabajo, objetivo general, objetivos específicos y una tabla para recoger la validación. Para determinar la confiabilidad del instrumento se aplicó el coeficiente Alfa de Crombachk debido a que el instrumento posee preguntas dicotómicas..., dando como resultado para la primera variables 0.82 considerando que el instrumento es confiable y recomienda su aplicación.

En lo que respecta al análisis de resultados, se consideró la representación en cuadros y gráficos estadísticos, permitiendo utilizar la estadística descriptiva para su interpretación mediante distribución de frecuencias absolutas y relativas ${ }^{(6)}$. Una vez realizados estos cálculos, se procedió a establecer si los porcentajes obtenidos para los factores y la variable siguen una distribución normal. Igualmente la información está representada en cuadros estadísticos, que permiten obtener resultados como base para dar las recomendaciones necesarias.

\section{RESULTADOS DE LA INVESTIGACIÓN}

Cuadro 1. Pacientes con enfermedades respiratorias, según edad, frecuencia y porcentaje hospital Dr. Rafael González Plaza 2008

\begin{tabular}{lllll}
\hline Edad & Fa & $\%$ & \\
\hline 15 a 22 años & 2 & 10 & \\
23 a 30 años & 3 & 15 & \\
Mayor de 30 años & 15 & 75 & \\
\hline Total & $\mathbf{2 0}$ & & $\mathbf{1 0 0}$
\end{tabular}

Fuente: Datos obtenidos por las autoras en el departamento de registros y estadísticas de Salud. 2008

Los datos proporcionados evidencian que durante el año 2008, se atendieron en el Hospital "Dr. González Plaza" del Municipio Naguanagua Estado Carabobo, un 10\% en edades comprendidas entre 15 a 22 años, un 15\% se encuentra en edades entre 23 y 30 años mientras que un $75 \%$ son personas mayores de 30 años.

Cuadro 2. Pacientes con enfermedades respiratorias, según sexo, frecuencia y porcentaje hospital Dr. Rafael González Plaza 2008

\begin{tabular}{lll}
\hline SEXO & Fa & $\%$ \\
\hline Masculino & 14 & 70 \\
Femenino & 6 & 30 \\
\hline Total & $\mathbf{2 0}$ & $\mathbf{1 0 0}$ \\
\hline
\end{tabular}

Fuente: Datos obtenidos por las autoras en el departamento de registros y estadísticas de Salud. 2008 
Los datos suministrados demuestran que durante el año 2008, se atendieron en el Hospital "Dr. González Plaza" del Municipio Naguanagua Estado Carabobo, un 70\% de usuarios del sexo masculino, mientras el restante $30 \%$ son de sexo femenino. Esto evidencia, que las enfermedades respiratorias son más frecuentes en los hombres.

Cuadro 3. Pacientes con enfermedades respiratorias, según procedencia, frecuencia y porcentaje hospital Dr. Rafael González Plaza 2008

\begin{tabular}{|c|c|c|}
\hline Procedencia & $\mathbf{F a}$ & $\%$ \\
\hline Carabobo & 16 & 80 \\
\hline Interior & 4 & 20 \\
\hline Otro & 0 & 0 \\
\hline Total & 20 & 100 \\
\hline
\end{tabular}

Fuente: Datos obtenidos por las autoras en el departamento de registros y estadísticas de Salud. 2008

Los datos proveídos evidencian que durante el año 2008, el promedio de procedencia de los pacientes con enfermedades respiratorias que se atendieron en el Hospital "Dr. González Plaza" es de $80 \%$ del Estado Carabobo y $20 \%$ del interior del país.

Cuadro 4. Pacientes con enfermedades respiratorias, según ocupación, frecuencia y porcentaje hospital Dr. Rafael González Plaza. 2008

\begin{tabular}{lll}
\hline Ocupación & Fa & $\%$ \\
\hline Del hogar & 4 & 20 \\
Empleado & 5 & 25 \\
Desempleado & 10 & 50 \\
Estudiante & 1 & 5 \\
Total & $\mathbf{2 0}$ & $\mathbf{1 0 0}$ \\
\hline
\end{tabular}

Fuente: Datos obtenidos por las autoras en el departamento de registros y estadísticas de Salud. 2008

Análisis: Los datos evidencian que del total de personas atendidas durante el año 2008, en el Hospital "Dr. González Plaza", un 50\% son desempleados, el $20 \%$ del hogar, un $25 \%$ empleado y un $5 \%$ restante son estudiantes.

Cuadro 5. Pacientes con enfermedades respiratorias, según tipo de enfermedad respiratoria, frecuencia y porcentaje hospital Dr. Rafael González Plaza. 2008

\begin{tabular}{llll}
\hline Tipo de Enfermedad Respiratoria & Fa & $\%$ & \\
\hline EPOC & 8 & 40 & \\
Bronquitis crónica & 8 & 40 & \\
TBC & 2 & 20 & \\
Enfisema & 0 & 0 & \\
\hline Total & $\mathbf{2 0}$ & & $\mathbf{1 0 0}$
\end{tabular}

Fuente: Datos obtenidos por las autoras en el departamento de registros y estadísticas de Salud. 2008 
Los datos proporcionados demuestran que durante el año 2008, se atendieron en el Hospital "Dr. González Plaza" un 40\% con EPOC, otro 40\% con problemas de Bronquitis crónica y un $20 \%$ con TBC.

\section{CONCLUSIONES}

La investigación tuvo como objetivo general Proponer un Modelo evaluativo del costo Fármaco Hospitalario de Pacientes con Enfermedades Respiratorias en el servicio de Hombres del Hospital Dr. Rafael González Plaza 1er trimestre del 2008. En relación con los objetivos específicos planteados en el presente estudio y a los resultados obtenidos se llegó a las diferentes conclusiones:

Referido a los datos demográficos de pacientes con EPOC se concluye que durante el año 2008, se atendieron en el Hospital "Dr. González Plaza" un 46\% de personas mayores de 67 años, de estos la mayoría se encuentra entre 57 y 67 años. Referido al sexo, un $60 \%$ de personas pertenecen al género femenino.

En función a los resultados obtenidos a través de la recolección de datos mediante el instrumento se llegan a las siguientes conclusiones, que sustentan el diagnóstico que dio lugar a la propuesta:

Referido a la morbilidad, se obtuvo que durante el año 2008 , se atendieron en el Hospital "Dr. González Plaza", un 40\% de pacientes con EPOC, proporción similar a la obtenida en el renglón Bronquitis crónica y un $20 \%$ con TBC. Referido a la edad predominante, fue la mayor de 30 años con un $75 \%$, un 15\% se encuentra en edades entre 23 y 30 años mientras que un $10 \%$ en edades comprendidas entre 15 a 22 años. De ellos, un $70 \%$ de los pacientes se ubican en el sexo masculino, mientras el restante $30 \%$ son de sexo femenino. Esto evidencia, que las enfermedades respiratorias son más frecuentes en los hombres mayores de 30 años.

Referido a la dimensión costo farmacológico, se tiene para el estrato Personal Administrativo (o) un promedio porcentual favorable de $55 \%$ por ciento en la alternativa siempre, casi siempre un 35 por ciento y por último en la alternativa nunca un 10 por ciento; mientras que la de los usuarios para la alternativa siempre un 20 por ciento, casi siempre 63 por ciento y nunca un 17 por ciento; lo que permite afirmar que dichos resultados son contrastantes para ambos estratos, ya que responden a una actitud ambivalente al tratar de establecer comparación entre las respuestas emitidas por los sujetos que conformaron la población en estudio; ya que a juicio de los usuarios, no se realizan acciones dirigidas a estimar el costo farmacológico, mientras que el personal administrativo, refiere que sí. Estos resultados evidencian que es necesario el diseño del modelo evaluativo del costo fármaco Hospitalario.

\section{Recomendaciones}

Según los resultados obtenidos en el desarrollo del presente trabajo investigativo, se formulan las siguientes recomendaciones las cuales se esperan sirvan de beneficio a la institución en donde se realizó el estudio y el estímulo en el avance de próximos trabajos de grado.

1. Presentar los resultados de la investigación a las autoridades de la institución donde se realizó la investigación, a fin de que sean tomados en cuenta para su análisis. 
2. En vista de que la mayor incidencia de enfermedad respiratoria se evidencia en mayores de 30 años, y de sexo masculino, se recomienda realizar estudios posteriores que determinen las causas asociadas a esta incidencia.

3. Revisar los estándares en atención al usuario que se manejan, a fin de mantener índices de mortalidad bajos.

4. Dado que la bronquitis crónica es la enfermedad respiratoria con mayor prevalecía, se recomienda orientar a los usuarios sobre los riesgos en la incidencia de los brotes de la misma, como son los períodos lluviosos, hábito tabaquito, entre otros.

5. A los estudiantes de enfermería del área de post grado, se les exhorta a conformar grupos de análisis sobre otras morbilidades, con la finalidad de mantenerse actualizados en las acciones gerenciales para el control de costos.

7. Realizar un análisis similar con otras patologías, a fin de realizar comparaciones con los resultados obtenidos.

\section{REFERENCIAS}

1. Morice, Ana y Achio, Mayra. (2003) Tendencias, costos y desafíos para la atención de las enfermedades crónicas en Costa Rica. Rev. cienc. adm. financ. segur. soc, vol.11, no.1, p.18-34. ISSN 1409-1259.

2. Organización Panamericana de Salud. Programa de Organización y Gestión de Sistemas y Servicios de Salud. División de desarrollo de sistemas y servicios de salud. Perfil del Sistema de Servicios de Salud de la Republica Bolivariana de Venezuela. Primera edición octubre de 1999. Segunda edición mayo de 2001.

3. Avila-Burgos L, Gutiérrez-Zúñiga C, Hernández-Peña P, Santos-Burgoa C, SilvaAyłaguer L. (1996) El costo social de la enfermedades respiratorias en la Ciudad de México: una experiencia piloto. Salud Publica Mex 1996;38:128-138.

4. Guyatt G, Berman L, Townsend L, Pugsley S, Chambers L. (1999) Medición de Calidad de vida a pacientes con enfermedades respiratorias . 42:773-778.

5. Arias, F. (2006) Proyecto de investigación. Introducción a la metodología científica. Quinta Edición. Caracas: Episteme

6. Hernández, Fernández y Baptista (2006) Metodología de la Investigación. Editorial Mac Graw Hill panamericana. Colombia. 


\section{ANEXO A}

INSTRUMENTOS PARA RECOLECTAR DATOS

CUESTIONARIO

\begin{tabular}{|c|c|c|c|}
\hline PARTE I . COSTOS FARMACOLOGICO & SIEMPRE & $\begin{array}{c}\text { CASI } \\
\text { SIEMPRE }\end{array}$ & NUNCA \\
\hline $\begin{array}{l}\text { 1. En la institución se le proporcionan los medicamentos } \\
\text { necesarios al usuario para el tratamiento de la enfermedad }\end{array}$ & & & \\
\hline $\begin{array}{l}\text { 2. El usuario ha tenido que comprara alguna medicina } \\
\text { indicada por el médico }\end{array}$ & & & \\
\hline $\begin{array}{l}\text { 3. El tiempo de hospitalización del usuario se ha prolongado } \\
\text { más del que consideraron al ingreso. }\end{array}$ & & & \\
\hline $\begin{array}{l}\text { 4. Las medicinas que le han proporcionado al usuario han sido } \\
\text { efectivas para la evolución de la enfermedad. }\end{array}$ & & & \\
\hline $\begin{array}{l}\text { 5. Como parte del tratamiento el usuario ha tenido que recibir } \\
\text { terapias de rehabilitación en clínicas privadas }\end{array}$ & & & \\
\hline $\begin{array}{l}\text { 6. El usuario Se ha tenido que realizar exámenes } \\
\text { complementarios en instituciones privadas. }\end{array}$ & & & \\
\hline $\begin{array}{l}\text { 7. Existe en la institución un Método de Estimación de costos } \\
\text { farmacológicos. }\end{array}$ & & & \\
\hline $\begin{array}{l}\text { 8. La institución cuenta con el personal requerido para la } \\
\text { estimación de los costos farmacológicos }\end{array}$ & & & \\
\hline $\begin{array}{l}\text { 9. Se mantiene un procedimiento estandarizado para el control } \\
\text { de los costos farmacológicos de hospitalización. }\end{array}$ & & & \\
\hline $\begin{array}{l}\text { 10. Se cuenta con los equipos tecnológicos para el control de } \\
\text { costo farmacológico. }\end{array}$ & & & \\
\hline 11. Están especificados los elementos del costo directo. & & & \\
\hline $\begin{array}{l}\text { 12. Existen formatos escritos para el registro de los elementos } \\
\text { del costo farmacológico. }\end{array}$ & & & \\
\hline $\begin{array}{l}\text { 13. Se realiza la verificación del costo farmacológico en que } \\
\text { se incurre en la institución. }\end{array}$ & & & \\
\hline
\end{tabular}


CUESTIONARIO DIRIGIDO AL USUARIO

\begin{tabular}{|l|l|l|l|l|l|l|}
\hline \multicolumn{5}{|c|}{ Parte I. DATOS SOCIODEMOGRÁFICOS } \\
\hline 1.Edad & \multicolumn{2}{|l|}{ 2.Sexo } & & 3.Procedencia & \multicolumn{2}{l|}{ 4.Ocupación } \\
\hline -Entre 15 y 22 años & & -Masculino & & -Carabobo & - Del hogar & \\
\hline -Entre 23 y 30 & & -Femenino & & -Interior & - Empleado & \\
\hline -Mayor de 30 & & & & -Otro & - Desempleado & \\
\hline & & & & & -Estudiante & \\
\hline
\end{tabular}

\begin{tabular}{|l|l|l|l|}
\hline \multicolumn{1}{|c|}{ PARTE II. COSTOS FARMACOLOGICO } & SIEMPRE & \multicolumn{1}{|c|}{ CASI } & NUNCA \\
\hline SIEMPRE & & & \\
\hline $\begin{array}{l}\text { 1. En la institución se le proporcionan los medicamentos } \\
\text { médico }\end{array}$ & & & \\
\hline $\begin{array}{l}\text { 3. El tiempo de hospitalización se ha prolongado más del que } \\
\text { consideraron al ingreso. }\end{array}$ & & & \\
\hline $\begin{array}{l}\text { 4. Las medicinas que le han proporcionado han sido efectivas } \\
\text { para la evolución de la enfermedad. }\end{array}$ & & & \\
\hline $\begin{array}{l}\text { 5. Como parte del tratamiento ha tenido que recibir terapias de } \\
\text { rehabilitación en clínicas privadas }\end{array}$ & & & \\
\hline $\begin{array}{l}\text { 6. Se ha tenido que realizar exámenes complementarios en } \\
\text { instituciones privadas. }\end{array}$ & & & \\
\hline
\end{tabular}

ISSN 1695-6141 